\title{
Experimental investigation of the heat transfer process between a tube bundle and an upward aqueous foam flow
}

\author{
J. Gylys ${ }^{1}$, T. Zdankus ${ }^{1}$, S. Sinkunas ${ }^{2}$, M. Babilas ${ }^{2} \&$ R. Jonynas ${ }^{2}$ \\ ${ }^{I}$ Energy Technology Institute, \\ Kaunas University of Technology, Lithuania \\ ${ }^{2}$ Department of Thermal and Nuclear Energy, \\ Kaunas University of Technology, Lithuania
}

\begin{abstract}
High heat transfer intensity and low energy consumption for coolant transportation to heat transfer location are really significant nowadays. An extended range of heat transfer intensity control (control of volumetric void fraction and flow rate of coolant) and low mass flow rate of coolant are one of the most important factors for heat exchangers. In some cases the usage of aqueous foam as a coolant can solve all of the mentioned problems. Our previous investigation showed that heat transfer between a heated tube and aqueous foam flow is over five to ten times lower for the water coolant, but the density of foam is more than one hundred times lower than that of water. When applying aqueous foam as a coolant in practice, some cases are problematic. The reasons for this are the variation of the structure and the characteristics of foam. Therefore, usage of aqueous foam as a coolant needs to be narrowly explored. This work follows our previous investigations. The tube bundle with a new arrangement of tubes was used in this research work. Spacing between the centres of the tubes in the horizontal lines of the bundle and the spacing between tube lines were equal to $0.03 \mathrm{~m}$. Each following line of tubes was located $0.01 \mathrm{~m}$ to the right of previous line. During the experimental investigation the dependence of heat transfer intensity (from tubes to foam flow) was determined on the volumetric void fraction of foam and the foam flow velocity. The experiments were performed for upward vertical foam flow for three different values of foam volumetric void fractions equal to $0.996,0.997$ and 0.998 . The velocity of the foam flow was changed from 0.14 to $0.32 \mathrm{~m} / \mathrm{s}$. The influence of tube position in the bundle for
\end{abstract}


the intensity of heat transfer was investigated. In research of the optimal geometry of a bundle, an average heat transfer intensity from bundle to foam flow was calculated and compared with the in-line and staggered tube bundles. The results showed that the heat transfer intensity of the investigated tube bundle to the upward foam flow is higher than that of the commonly-used heat exchangers. The analysis of the results is also presented in this paper.

Keywords: tube bundle, aqueous foam, upward flow, heat transfer.

\section{Introduction}

Heat transfer between heated surfaces and two-phase systems (such as aqueous foam) is an area still studied on the small scale. In our previous investigations, conditions of foam flow for creating the proper and steady heat transfer process were defined [1].

The characteristic of aqueous foam, which is named statically stable foam, demonstrates its perfect application for the heat transfer process. Certainly, this statically stable foam keeps its initial structure and dimensions of bubbles for a conditionally long interval of time - from several minutes to several hours even after the foam generation terminates $[1,2]$. Therefore, this is a reason for using this type of coolant as the heat transfer working fluid in our investigation.

Heat transfer between a single cylindrical surface-tube (as well as a tube line) and upward directed aqueous foam flow was investigated in our previous works. It was noticed that the value of the heat transfer coefficient for a lone heated tube to foam flow was 21-42 times higher than that of air (single-phase fluid flow) and 6-11 times less than that of water flow (the velocity of water flow was the same as for foam and air). The foam flow velocity was equal to $0.4 \mathrm{~m} / \mathrm{s}$ and the volumetric void fraction of the foam varied from 0,996 to 0,998 for the above mentioned case. For comparison, the density of the same coolants was equal: 1.2 $\mathrm{kg} / \mathrm{m}^{3}$ for air; $3.2-5.2 \mathrm{~kg} / \mathrm{m}^{3}$ for foam; $998.2 \mathrm{~kg} / \mathrm{m}^{3}$ (190-310 times more than the density of aqueous foam) for water.

Applying the two-phase coolants, such as aqueous foam, in practice could significantly reduce material and energy demands, simultaneously sustaining the proper heat transfer intensity on heated surfaces. Moreover, the two-phase foam coolant (vs. single-phase) has the additional possibility of increasing the intensity of heat transfer by changing the volumetric void fraction of foam. Such advantages of aqueous foam give a chance to create a compact, light, safe and economic heat exchanger.

However, it was also found before that the specific foam properties [3-5] determined other consistent patterns of tube bundle cooling. Therefore, we performed the investigation where heat transfer between the staggered [6] and inline $[7,8]$ tube bundles and the vertically upward (in one case) and downward (in the other case) after 180 degree turning foam flow was explored. The purpose for this is acquiring the data that could be used for designing the real heat exchanger.

The major objectives of our research are to determine and estimate the influence of tube bundle types and the geometry of the intensity of tube bundle heat transfer to foam flow. It is well-known that bundles (behaving as obstacles 
in a flow) induce substantial changes in foam structure: the current bubbles are transformed by collapsing, dividing and appearing as new ones. With the help of gravity and capillary forces, these transformations facilitate the liquid drainage from the foam and affect the liquid film's thickness, composing on the heated surfaces and channel walls. Such effects can significantly either increase or decrease the heat transfer rates.

One of the main objectives for this investigation is also to determine the optimal type of tube bundle that could guarantee the heat transfer to two-phase aqueous foam flow at the maximum level. The dependence of tubes of the nonstandard tube bundle heat transfer intensity on foam flow velocity and volumetric void fraction are determined and discussed in this work. Furthermore, the influence of tube position in the bundle was also investigated.

Further researches are planned to be organised for estimating the optimal type and geometry of tube bundle that maximally increases the intensity of heat transfer to the foam flow.

\section{Experimental set-up}

The experimental equipment [7, 9] consisted of the following parts: a vertical experimental channel with integrated non-standard tube bundle, gas and liquid control valves, gas and liquid flow meters, a liquid storage reservoir, a liquid level control reservoir, a compressor with a receiver, an electric current transformer and a stabilizer. The cross section of the experimental channel had the dimensions of $0.14 \times 0.14 \mathrm{~m}^{2}$; its height was $1.8 \mathrm{~m}$. The walls of the channel were made from transparent material so that the foam flow could be observed visually.

Statically stable foam - one type of aqueous foam - was used as a coolant for the experiments. Statically stable foam flow was generated from the water solution with detergents. The concentration of detergents was constant at $0.5 \%$ level in all the experiments. The solution (water-detergent) was supplied from the reservoir onto the special perforated plate. The foam flow was generated during gas and liquid contact. The foam flow parameters control was fulfilled using gas and liquid valves.

The perforated stainless steel plate with a thickness of $2 \mathrm{~mm}$ for foam generation was installed at the upside of the experimental channel; orifices were located in a staggered order with diameter equal to $1 \mathrm{~mm}$ and $5 \mathrm{~mm}$ spacing between the centres of the holes.

A non-standard tube bundle (Fig. 1, a) was constructed out of seven rows of tubes (diameter $d=0.02 \mathrm{~m}$, amount of tubes in a row: $1^{\text {st }}=5,2^{\text {nd }}=4,3^{\text {rd }}=4,4^{\text {th }}=$ $5,5^{\text {th }}=4,6^{\text {th }}=4,7^{\text {th }}=5$ ). The spaces between the centres of tubes in a row across the tube bundle were $s_{I}=0.03 \mathrm{~m}$ and the distance between the axis piloted out through the tubes centres in horizontal rows was $s_{2}=0.03 \mathrm{~m}$. The tubes in a second row were moved into the right-hand side considering the first row with a distance $s_{3}=0.01 \mathrm{~m}$. The third row of tubes were moved with the same distance $s_{3}=0.01 \mathrm{~m}$ to the right-hand side regarding the second row of tubes. The fourth row of tubes was aligned horizontally the same way as the first. The fifth, sixth 
and seventh rows were stated complexly like a mirror-image of the first three rows. Due to this kind of complicated array of tubes, the tube bundle was named "non-standard".

Tubes of the in-line tube bundle (Fig. 1b) were located in six rows of tubes with five tubes in each of them. Spacing between tubes in rows was $s_{I}=0.03 \mathrm{~m}$ and spacing between rows of the tubes was $s_{2}=0.03 \mathrm{~m}$ too.

A one tube-calorimeter was heated electrically. This tube was made of copper and had an external diameter of $0.02 \mathrm{~m}$. The ends of the heated tube were sealed and insulated to prevent heat loss through them. During the experiments, the position of the calorimeter alternated and one tube in the bundle was replaced with a calorimeter. The tubes located by the walls of the channel were not investigated. An electric current value of the heated tube was measured by an ammeter and the voltage was measured by a voltmeter. The temperature of the calorimeter surface was measured by eight calibrated thermocouples: six of them were placed around the central part of the tube and two of them were placed in both sides of the tube with a distance of $50 \mathrm{~mm}$ from the central part. The temperature of the foam flow was measured by two calibrated thermocouples: one in front of the bundle and one behind it.

Measurement accuracies for flows, temperatures and heat fluxes were of the range correspondingly $1.5 \%, 0.15-0.20 \%$ and $0.6-6.0 \%$.

During the experimental investigation a relationship was obtained between an average heat transfer coefficient $h\left(N u_{f}\right)$ from one side and a foam flow volumetric void fraction $\beta$ and gas flow Reynolds number $R e_{g}$ from the other side:

a)

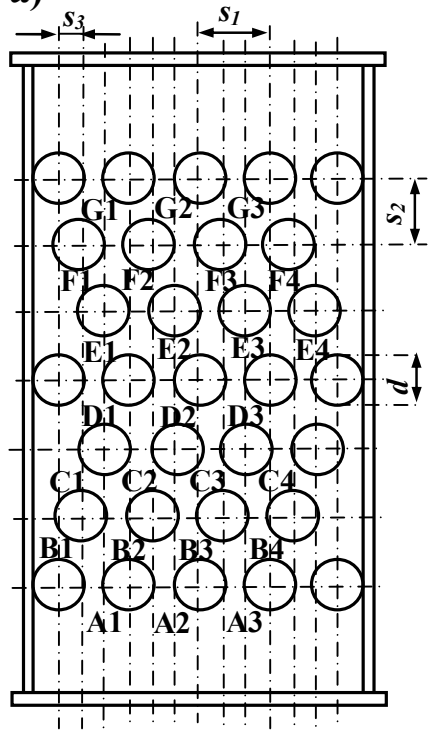

b)

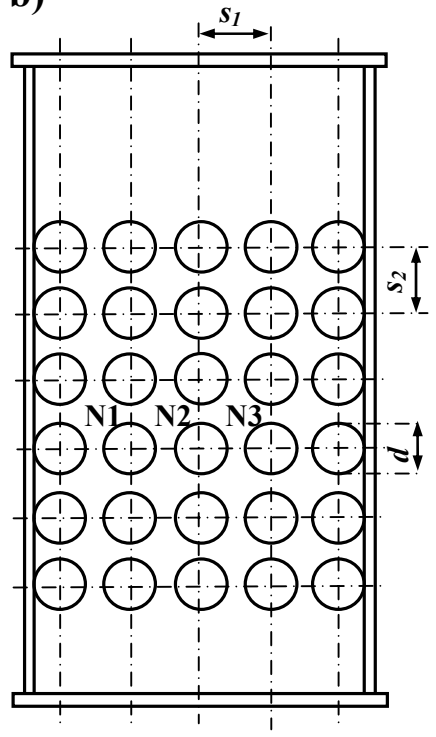

Figure 1: $\quad$ Non-standard (a) and in-line (b) tube bundles in upward foam flow. 


$$
N u_{f}=f\left(\beta, \operatorname{Re}_{g}\right) .
$$

The Nusselt number was computed by the formula

$$
N u_{f}=\frac{h d}{\lambda_{f}} .
$$

Here $\lambda_{f}$ is the thermal conductivity of the statically stable foam flow, $\mathrm{W} /(\mathrm{m} \cdot \mathrm{K})$, computed by the equation

$$
\lambda_{f}=\beta \lambda_{g}+(1-\beta) \lambda_{l} .
$$

An average heat transfer coefficient was calculated as

$$
h=\frac{q_{w}}{\overline{\Delta T}} .
$$

The gas Reynolds number of foam flow was computed by the formula

$$
\operatorname{Re}_{g}=\frac{G_{g} d}{A v_{g}} .
$$

The foam flow volumetric void fraction was expressed by the equation

$$
\beta=\frac{G_{g}}{G_{g}+G_{l}} .
$$

Experiments were performed within the limits of Reynolds number diapason for gas $\left(R e_{g}\right)$ : 190 440 (laminar flow regime) and foam volumetric void fraction $(\beta)$ : $0.996 \sim 0.998$. The gas velocity for foam flow was changed from 0.14 to $0.32 \mathrm{~m} / \mathrm{s}$.

\section{Results}

The heat transfer from the in-line tube bundle (Fig. 1b) to the vertical upward foam flow was investigated initially $[8,9]$. Then the non-standard tube bundle (Fig. 1a) was installed in an experimental channel and the heat transfer process between tubes of the non-standard tube bundle and the upward vertical foam flow was investigated.

The comparison of the heat transfer intensity of the tubes A2 and D2 from the non-standard tube bundle to the upward foam flow for $\beta=0.996 \sim 0.998$ and $R e_{g}=190 \sim 440$ is shown in Fig. 2.

With increasing foam flow gas Reynolds number $R e_{g}$ from 190 to 440 heat transfer intensity $\left(N u_{f}\right)$ of the tube A2 (the middle tube of the frontal line of the non-standard tube bundle) to the upward foam flow increases by 2.8 times (from $N u_{f}=450$ to $N u_{f}=1273$ ) for foam volumetric void fraction $\beta=0.996$, by 2.6 times for $\beta=0.997$ (from $N u_{f}=372$ to $N u_{f}=977$ ), and by 2.4 times for $\beta=0.998$ (from $N u_{f}=285$ to $N u_{f}=697$ ). The heat transfer intensity of tube A2 is on average 1.7 times higher for the wettest foam flow $(\beta=0.996)$ in comparison to the drier foam flow $(\beta=0.997)$ and 2.2 times higher in comparison with the driest foam flow $(\beta=0.998)$. 


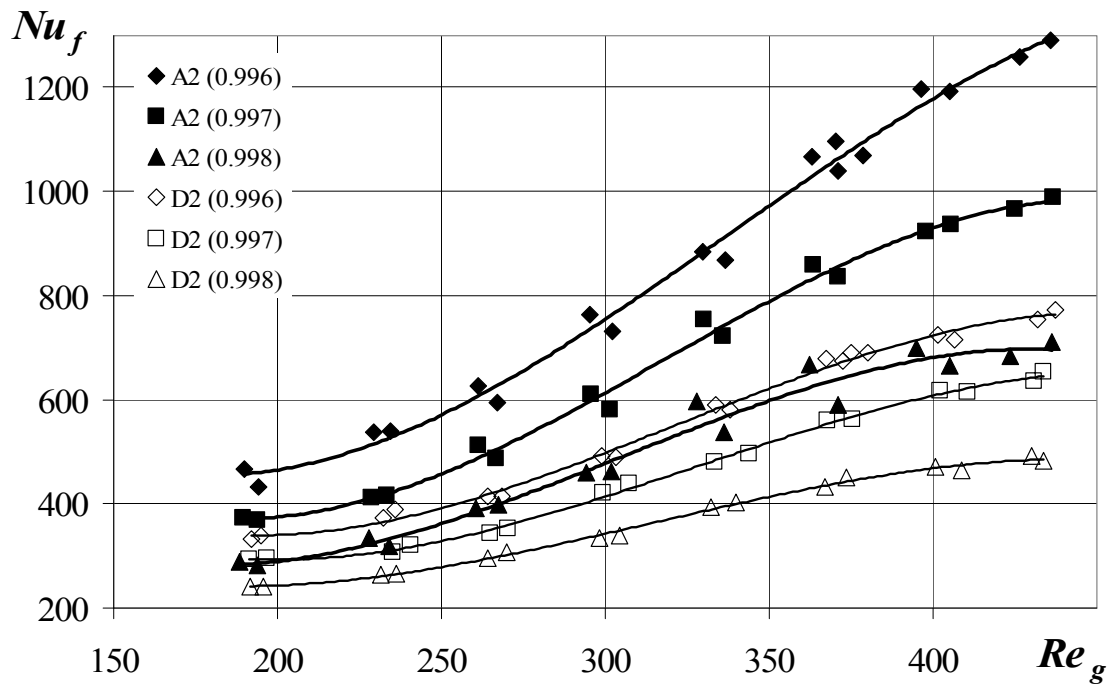

Figure 2: The comparison of the heat transfer intensity of tubes A2 and D2 of the non-standard tube bundle to the upward foam flow, $\beta=0.996$, 0.997 and 0.998 .

The heat transfer intensity $\left(N u_{f}\right)$ of tube D2 (the middle tube of the fourth line of the non-standard tube bundle) to the upward foam flow increases by 2.3 times (from $N u_{f}=335$ to $N u_{f}=764$ ) for the wettest foam flow $(\beta=0.996)$, and by 2.2 times for the foam flow with $\beta=0.997$ (from $N u_{f}=295$ to $N u_{f}=644$ ), and twice for the foam flow with $\beta=0.998$ (from $N u_{f}=241$ to $N u_{f}=487$ ) for $R e_{g}=190 \sim 440$. The heat transfer intensity of tube D2 is on average 1.4 times higher for the wettest foam flow $(\beta=0.996)$ in comparison to the drier foam flow $(\beta=0.997)$ and 1.7 times higher in comparison to the driest foam flow $(\beta=0.998)$.

The heat transfer intensity of tube A2 is on average 1.6 times higher than that of tube D2 for $\beta=0.996,1.5$ times higher than that of tube D2 for $\beta=0.997$ and 1.4 times higher than that of tube $\mathrm{D} 2$ for $\beta=0.998,\left(R e_{g}=190 \sim 440\right)$.

The geometry of the non-standard tube bundle acts on the heat transfer intensity of the tubes located at the different places in the further horizontal tube lines of the non-standard tube bundle to upward foam flow (Fig. 3). Tubes D1 and D3 are located in the bundle at the same distance from the centre of the experimental channel, but the heat transfer intensity of these tubes is not the same (Fig. 3). The heat transfer intensity of tube D1 is highest, the heat transfer intensity of tube D3 is less than that of the tube D1 and the heat transfer intensity of tube D2 is almost the same as that of tube D3. The heat transfer intensity of tube D1 is on average $18 \%$ higher than for tubes D3 and D2 for $\beta=0.997$ and $R e_{g}=190 \sim 440$.

In the case of the in-line tube bundle (Fig. 1b) the situation was different. Tubes N1 and N3 were located in the bundle at the same distance from the centre 


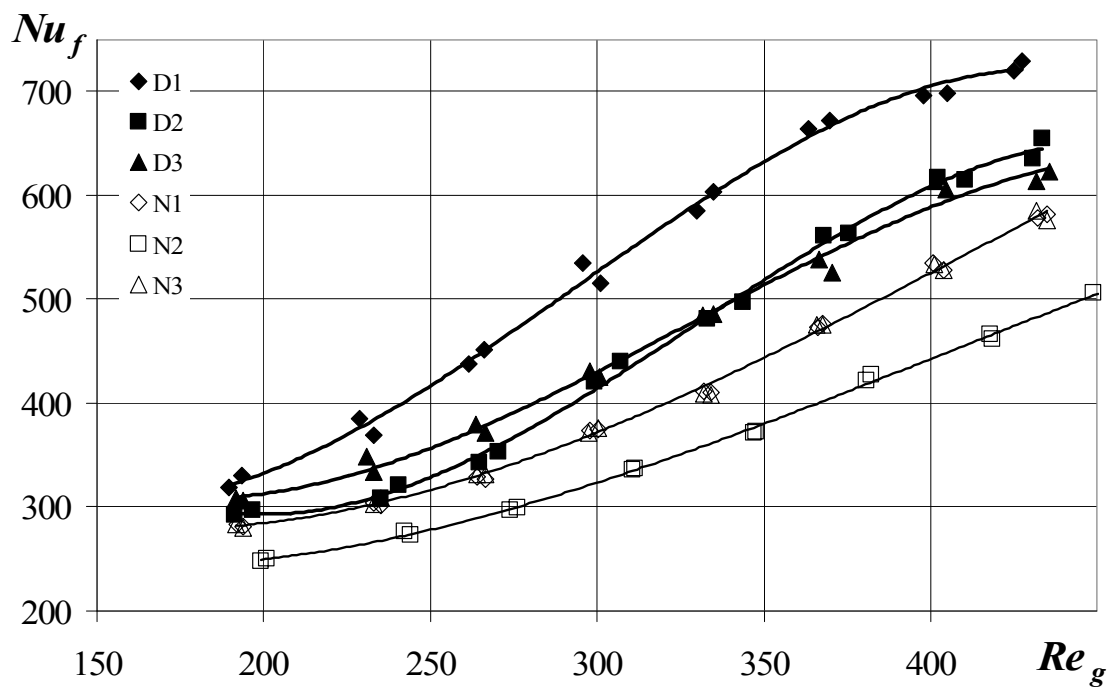

Figure 3: The heat transfer intensity of tubes D1, D2 and D3 (tubes of the non-standard tube bundle) and N1, N2 and N3 (tubes of the in-line tube bundle) to the upward foam flow, $\beta=0.997$.

of the experimental channel and the heat transfer intensity of these tubes was identical to (Fig. 3) or higher than the heat transfer intensity of tube N2, which is located in the middle of the fourth line of tubes.

The comparison of the heat transfer intensity of tubes D2 (of the non-standard tube bundle) and N2 (of the in-line tube bundle $1.5 \times 1.5$ ) to the upward foam flow at the volumetric void fraction $\beta=0.996,0.997$ and 0.998 is shown in Fig. 4. The heat transfer intensity of tube D2 is higher than that of tube B4 on average by 1.34 times for $\beta=0.996$ and by 1.25 times for $\beta=0.997$ and $0.998\left(\operatorname{Re}_{g}=190 \sim 440\right)$. This phenomenon can be explained by more intensive turbulence of foam flow in the case of the non-standard tube bundle.

The experimental results of investigation of the heat transfer from the nonstandard tube bundle to the upward foam flow were generalized by criterion equation (7) using dependence between Nusselt number $N u_{f}$ and gas Reynolds $R e_{g}$ number. This dependence within the interval $190<R e_{g}<440$ for the nonstandard tube bundle in the upward foam flow with the volumetric void fraction $\beta=0.996,0.997$, and 0.998 can be expressed as follows:

$$
N u_{f}=c \beta^{n} \operatorname{Re}_{g}^{m} .
$$

For tube D1 in the upward foam flow: $c=0.75, n=-233, m=20.9(1.042-\beta)$.

For tube D2 in the upward foam flow: $c=2.34, n=275, m=82.4(1.010-\beta)$.

For tube D3 in the upward foam flow: $c=12.80, n=518, m=127.3(1.004-\beta)$. 


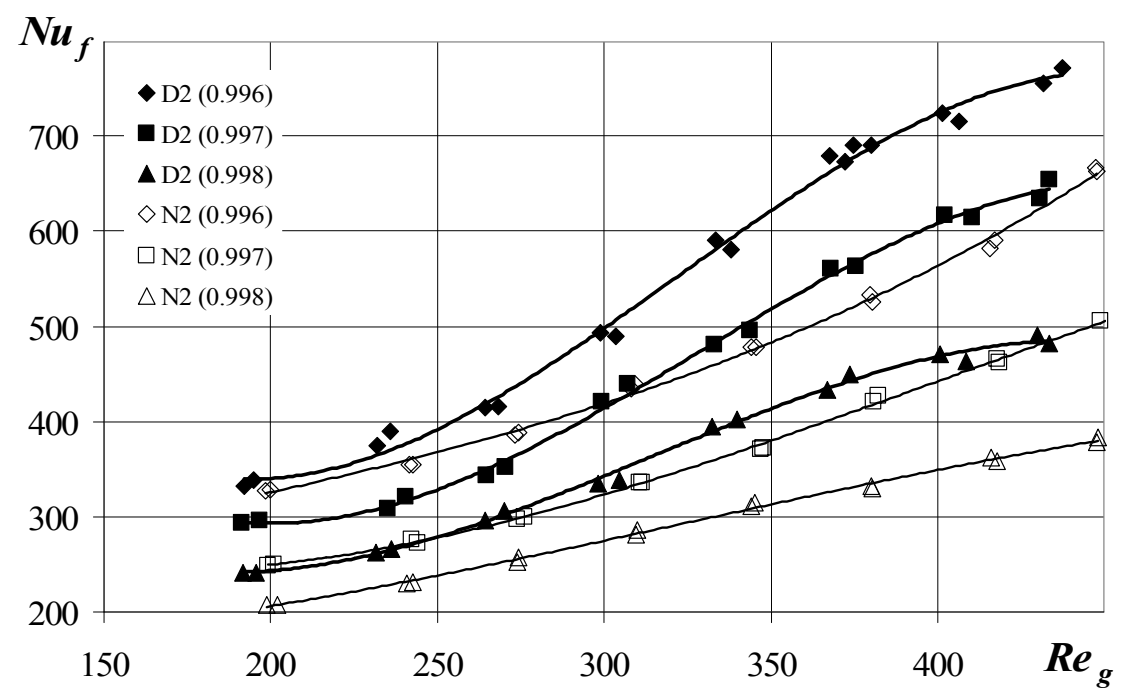

Figure 4: The heat transfer intensity of tubes D2 and N2 from the fourth lines of the non-standard and in-line bundles to the upward foam flow; $\beta=0.996,0.997$ and 0.998 .

\section{Conclusions}

The heat transfer process between the tubes of the non-standard tube bundle and the upward statically stable foam flow was investigated experimentally.

The heat transfer intensity of the side tubes N1 and N3 (the fourth line of the in-line tube bundle $1.5 \times 1.5)$, which were located at the same distance from the centre of the channel, to the upward foam flow was identical to or higher than that of the middle tube $\mathrm{N} 2$.

The heat transfer intensity of the side tubes D1 and D3 (the fourth line of the non-standard tube bundle), which were located at the same distance from the centre of the channel, to the upward foam flow was found to be different. The heat transfer intensity of tube D1 was determined to be higher than that of tube D3. The heat transfer intensity of tubes D2 and D3 was almost the same for $\beta=0.997$.

The heat transfer intensity of the tubes from the fourth horizontal tubes' line in the non-standard tube bundle is higher than that of the tubes in the in-line tube bundle $1.5 \times 1.5$.

The criterion equation (7) should be applied for calculating the heat transfer intensity of tubes from further lines of non-standard tube bundles to the upward vertical statically stable foam flow. 


\section{Nomenclature}

$A$ - cross section area of experimental channel, $\mathrm{m}^{2} ; c, m, n$ - coefficients; $d-$ outside diameter of tube, m; $G$ - volumetric flow rate, $\mathrm{m}^{3} / \mathrm{s} ; \mathrm{Nu}$ - Nusselt number; $q$ - heat flux density, $\mathrm{W} / \mathrm{m}^{2} ; R e$ - Reynolds number; $\bar{T}-$ average temperature, $\mathrm{K} ; h$ - average coefficient of heat transfer, $\mathrm{W} /\left(\mathrm{m}^{2} \cdot \mathrm{K}\right) ; \beta-$ volumetric void fraction; $\lambda$ - thermal conductivity, $\mathrm{W} /(\mathrm{m} \cdot \mathrm{K}) ; v-$ kinematic viscosity, $\mathrm{m}^{2} / \mathrm{s}$.

\section{Indexes}

$f$-foam;

$g$ - gas;

$l$ - liquid;

$w$ - wall of heated tube.

\section{References}

[1] Gylys, J., Hydrodynamics and Heat Transfer under the Cellular Foam Systems, Technologija: Kaunas, 1998.

[2] Tichomirov V., Foams. Theory and Practice of Foam Generation and Destruction, Chimija: Moscow, 1983.

[3] Sadoc, J. F., Rivier, N., Foams and Emulsions, Nato ASI Series, 1997.

[4] Nguyen A. V., Liquid Drainage in Single Plateau Borders of Foam. Journal of Colloid and Interface Science, 249(1), pp. 194-199, 2002.

[5] Garrett P. R, Recent developments in the understanding of foam generation and stability. Chemical Engineering Science, 48(2), pp. 367-392, 1993.

[6] Gylys, J., Sinkunas, S., Zdankus, T., Analysis of staggered tube bundle heat transfer to vertical foam flow, J. International Journal of Heat and Mass Transfer, 51(1-2), pp. 253-262, 2008.

[7] Gylys J., Zdankus T., Sinkunas S., Giedraitis V., Study of In-line Tube Bundle Cooling in Vertical Foam Flow. WSEAS Transactions on Heat and Mass Transfer, Iss. 6, 1, pp. 632-637, 2006.

[8] Gylys, J.; Sinkunas, S.; Zdankus, T.; Giedraitis, V., Balcius, A., Foam flow turn influence on the in-line tube bundle heat transfer intensity, Computational Methods and Experimental Measurements XIII, Prague, Czech Republic, pp. 457-464, 2007.

[9] Gylys, J.; Zdankus, T.; Gabrielaitiene, I., Sinkunas, S.; Experimental research of heat transfer from an in-line tube bundle to a vertical foam flow, J.: Heat Transfer Research, 40(1), pp. 455-472, 2009. 